\title{
Post-translational modifications of Kaposi's sarcoma-associated herpesvirus regulatory proteins - SUMO and KSHV
}

\author{
Mel Campbell and Yoshihiro Izumiya* \\ Department of Dermatology, University of California Davis, Sacramento, CA, USA
}

\section{Edited by:}

Keiji Ueda, Osaka University Graduate

School of Medicine, Japan

Reviewed by:

Keiji Ueda, Osaka University Graduate

School of Medicine, Japan

Masahiro Fujimuro, Kyoto

Pharmaceutical University, Japan

\section{*Correspondence:}

Yoshihiro Izumiya, Department of Dermatology, University of California

Davis Cancer Center, University of

California Davis, Research III Room

2400, 4645 2nd Avenue, Sacramento,

CA 95817, USA.

e-mail: yizumiya@ucdavis.edu
KSHV latency can be envisioned as an outcome that is balanced between factors that promote viral gene expression and lytic replication against those that facilitate gene silencing and establish or maintain latency. A large body of work has focused on the activities of the key viral regulatory proteins involved in KSHV latent or lytic states. Moreover, recent studies have also begun to document the importance of epigenetic landscape evolution of the KSHV viral genome during latency and reactivation. However, one area of KSHV molecular virology that remains largely unanswered is the precise role of post-translational modifications on the activities of viral factors that function during latency and reactivation. In this review, we will summarize the post-translational modifications associated with three viral factors whose activities contribute to the viral state. The viral proteins discussed are the two major KSHV encoded transcription factors, K-Rta (KSHV replication and transcriptional activator) and K-bZIP (KSHV basic leucine zipper) and the viral latency-associated nuclear antigen (LANA). A special emphasis will be placed on the role of the sumoylation pathway in the modulation of the KSHV lifecycle. Newly uncovered small ubiquitin-like modifier (SUMO)-associated properties of LANA and K-Rta will also be presented, namely LANA histone targeting SUMO E3 ligase activity and K-Rta SUMO-targeted ubiquitin ligase function.

\section{Keywords: KSHV, LANA, K-bZIP, K-Rta, SUMO, post-translational modification, transcription}

\section{INTRODUCTION}

Reactivation from a latent state is an important feature of infection and disease caused by many herpesviruses. Although the molecular mechanisms of reactivation have been extensively studied, gaps exist in our current knowledge concerning the processes by which these viruses establish, maintain, and emerge from latency. KSHV latency can be envisioned as the outcome balanced between factors that require viral gene expression and lytic replication against those that are essential for establishment and/or maintenance of latent infection. In this review, we will provide an update on three viral factors whose activities contribute to the viral state with an emphasis on post-translational modifications that modulate multiple functions of these factors. The viral proteins discussed are the two major KSHV encoded transcription factors, K-Rta and K-bZIP and the viral latency-associated nuclear antigen (LANA).

\section{K-bZIP}

K-bZIP (K8) is an early lytic gene and is among the earliest viral genes expressed after acute infection or during reactivation from latency. K-bZIP is a 237 -amino acid protein containing a basic and leucine zipper domain and is the structural and positional analog of Epstein-Barr virus (EBV) BZLF1 (Lin et al., 1999). K-bZIP has been reported to be phosphorylated (Polson et al., 2001), sumoylated (Chang et al., 2010; Lefort et al., 2010), and acetylated (Lefort et al., 2010). K-bZIP directly associates with K-Rta in KSHV infected cells and represses K-Rta-mediated transactivation at a subset of KSHV promoters. This activity of K-bZIP is subject to post-translational modulation via phosphorylation (Izumiya et al., 2007) and sumoylation (Izumiya et al., 2005). As detailed below, K-bZIP repression activity on $\mathrm{K}$-Rta-mediated transactivation is regulated in an opposing manner by these two post-translational modifications. While K-bZIP repression is largely dependent on sumoylation, phosphorylation serves as a negative regulator. A schematic diagram of $\mathrm{K}$ bZIP and its post-translational modification sites are presented in Figure 1.

\section{PHOSPHORYLATION}

K-bZIP was reported to be phosphorylated on residues Thr 111 and Ser 167 (Polson et al., 2001; Izumiya et al., 2007). Interestingly, these sites are contained within cellular cyclin-dependent kinase (CDK) recognition sites with the consensus sequence (S/T)PXR suggesting that K-bZIP may be phosphorylated by CDKs. Indeed, K-bZIP was confirmed to be a substrate for several cellular CDKcyclin complexes in vitro, including CDK-2/cyclin A, CDK-1/cyclin B, and CDK-2/cyclin E (Polson et al., 2001). K-bZIP was also found to interact with, and be a substrate for, the viral protein kinase (vPK, ORF36). vPK is a serine/threonine protein kinase expressed from mRNAs with early and early-late kinetics and the major vPK phosphorylation site on K-bZIP was determined to also be residue threonine (T) 111 (Izumiya et al., 2007). K-bZIP 


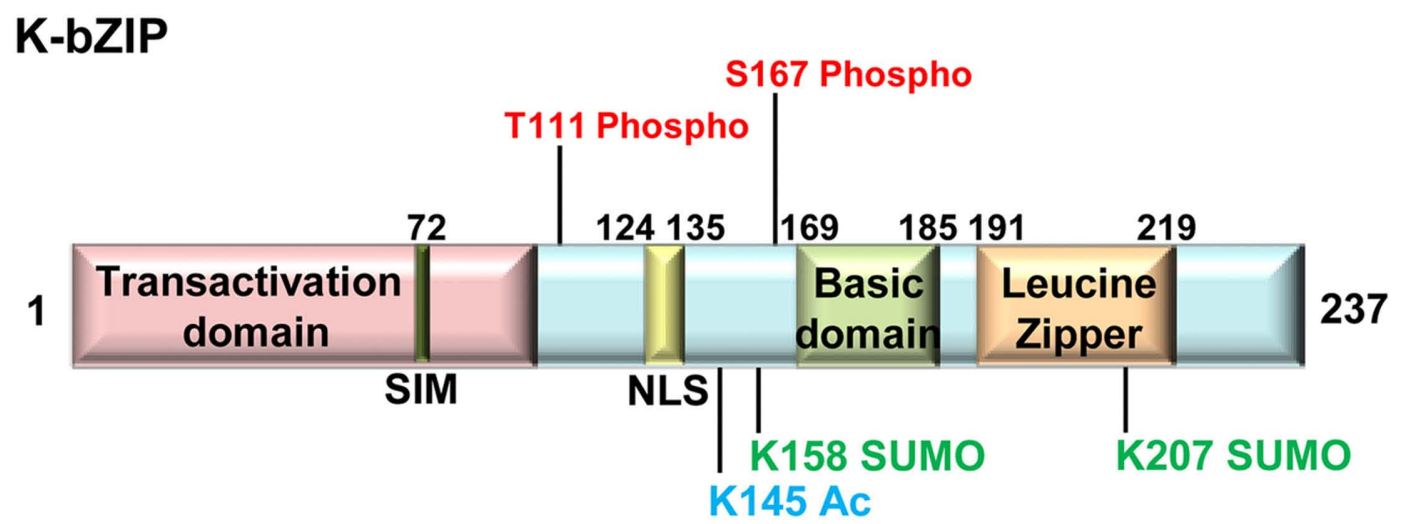

FIGURE 1 | Schematic representation of KSHV K-bZIP. K-bZIP protein and its post-translational modification sites as discussed in the text are depicted. Phosphorylation (Phospho), acetylation (Ac), and sumoylation
(SUMO) sites are shown. The K-bZIP SUMO interaction motif (SIM) and nuclear localization signal (NLS) are also indicated. Numbers indicate amino acid position. directly interacts with K-Rta and represses K-Rta activation of certain K-Rta responsive promoters such as ORF57 and K-bZIP, but not at other similarly responsive promoters such as PAN. The functional significance of K-bZIP T111 phosphorylation site with respect to repression of K-Rta-mediated transactivation was examined using a mutagenesis strategy in which T111 was changed to a non-phospho acceptor reside alanine, or to aspartic acid, a phospho-mimetic. In co-transfection experiments with K-Rta and a K-Rta responsive promoter construct, K-bZIP-T111D repressive function was similar to wild-type K-bZIP, whereas K-bZIP-T111A was a more potent repressor. Moreover, K-bZIP-T111A was found to be more heavily sumoylated than wild-type K-bZIP or K-bZIPT111D and overexpression of $\mathrm{vPK}$ reduced the sumoylation of wild type K-bZIP. Taken together, these results are consistent with a model whereby phosphorylation at T111 has a negative effect on both the extent of sumoylation and the repressive activity of K-bZIP (Izumiya et al., 2007). Thus K-bZIP activities are modulated by phosphorylation and sumoylation in an antagonistic fashion similar to other cellular transcription factors such as ELK1 (Girdwood et al., 2003), AP-1 (Bossis et al., 2005), AIB1 (Wu et al., 2006), progesterone receptor B (PR-B; Daniel et al., 2007; Daniel and Lange, 2009), STAT1 (Zimnik et al., 2009), and KAP-1 (Goodarzi et al., 2011). KSHV genome-wide reporter assays support the notion that K-bZIP preferentially represses early-lytic genes but not late gene promoters (Ellison et al., 2009), consistent with temporal regulation of viral gene expression, potentially mediated, in part, through a K-bZIP SUMO-phospho switch.

\section{ACETYLATION}

K-bZIP was reported to be acetylated on multiple residues and K145 was identified as one acetylation site (Lefort et al., 2010). KbZIP was previously found associated with CBP (Hwang et al., 2001) and the ability of K-bZIP to repress transcription was reported to be mediated through sequestration of CBP. In cotransfection experiments, increasing amounts of CBP resulted in increased acetylation of K-bZIP and a reversal of K-bZIP-mediated repression of the IFN $\alpha$-activated $2^{\prime}, 5^{\prime}$-OAS gene. In addition to effects on chromatin, CBP may be capable of acetylating K-bZIP.
However, the functional role of K-bZIP acetylation in the KSHV life cycle remains to be seen.

\section{SUMOYLATION}

Sumoylation (small ubiquitin-like modifier) was identified as a reversible post-translational protein modification in 1997 (reviewed in Geiss-Friedlander and Melchior, 2007). Three isoforms of SUMO exist: SUMO-1, -2, and -3. SUMO-2 and SUMO-3 are highly homologous ( $97 \%$ identity in the mature form), whereas SUMO-1 is more distantly related to both $(\sim 50 \%)$. Hundreds of protein targets for SUMO-modification have been identified, and the majority of these are nuclear proteins. Similar to phosphorylation, sumoylation is a rapid and reversible modification. Moreover, in a manner similar to the binding of phosphorylated tyrosine residues by signaling molecules carrying phosphotyrosine (PTB) or Src homology 2 (SH2) domains, sumoylated proteins are specifically recognized by proteins containing one or more SUMOinteracting motifs (SIM). Analogous to ubiquitylation, conjugation of SUMO to target proteins is a multi-step process involving an E1 activating enzyme heterodimer SAE1/SAE2 (AOS1/UBA2), an E2 conjugating enzyme Ubc9 (ubiquitin-like protein SUMO1 conjugating enzyme 9), and an E3 ligase, which is believed to provide specificity within the sumoylation pathway (GeissFriedlander and Melchior, 2007). Sumoylation is a highly dynamic process, and its outcomes are very diverse, ranging from changes in subcellular localization to altered activity and, in some cases, stability of the modified protein. The underlying principle of sumoylation is the alteration of inter- or intra-molecular interactions of the modified substrate (Prudden et al., 2007; Uzunova et al., 2007; Xie et al., 2007). For DNA tumor viruses, the immediate-early and early gene products, which are transcriptional factors, are often sumoylated. Examples include IE1 (immediate-early 1 protein) and IE2 (immediate-early 2 protein) of cytomegalovirus (CMV; Hofmann et al., 2000; Spengler et al., 2002; Hsu et al., 2004), E1 and E2 of human papillomavirus (HPV; Rosas-Acosta et al., 2005; Wu et al., 2007), BZLF1 of EBV (Adamson and Kenney, 2001), K-bZIP, and LANA2 of KSHV (Izumiya et al., 2005; Marcos-Villar et al., 2009). Viral proteins are also known to modulate the sumoylation 
of specific cellular proteins. For example, HPV E7 protein and adenovirus E1A protein block sumoylation of RB (Ledl et al., 2005) and KSHV vPK inhibits the sumoylation of KAP-1 (Chang et al., 2009). Viruses can also affect global sumoylation of cellular proteins by directly modulating the SUMO machinery. Avian Adenovirus Gam1 is known to inhibit the SUMO E1 activating enzyme by targeting the SAE1/SAE2 (AOS1/UBA2) heterodimer to cullin RING ligases (CRLs) and promoting SAE1 ubiquitylation and degradation (Colombo et al., 2002; Boggio et al., 2007).

The repressive activity of K-bZIP requires sumoylation at lysine-158 and an intact leucine zipper region. Sumoylation at this site is required for the association of K-bZIP with the cellular E2 SUMO conjugation enzyme Ubc9. Both SUMO-1 and SUMO$2 / 3$ were found conjugated to a significant fraction $(\sim 10 \%)$ of endogenous K-bZIP in BCBL-1 cells. Mutation of lysine-158 to arginine eliminated SUMO-modification of K-bZIP in vivo with a concomitant loss of the majority of K-bZIP repressive function. Although SUMO-modification of K-bZIP may influence its repressive function through several mechanisms, including effects on the physical interaction between K-bZIP and K-Rta, it is likely that a major effect of SUMO is mediated by its ability to recruit Ubc9 to K-bZIP target promoters. Ubc9 binding to K-bZIP, as well as co-occupancy of K-bZIP, K-Rta, and SUMO at target viral promoters has been observed. Based on these results, we predicted that K-bZIP may function as a SUMO E3 ligase or SUMO adaptor which functions to deliver Ubc9 to potential substrates (Izumiya et al., 2005). In addition to lysine-158, Lefort et al. (2010) have also identified a previously unrecognized sumoylation site in a K-bZIP splice variant (K207).

Subsequently, Chang et al. (2010) have confirmed that K-bZIP functions as the prototypical viral SUMO E3 ligase. K-bZIP was found to be a SIM-containing poly-SUMO-specific E3 ligase with specificity for SUMO-2/3. As discussed above, K-bZIP had been previously known to associate with Ubc9 (Izumiya et al., 2005), Chang et al. further demonstrated that K-bZIP bound SUMO-2 and SUMO-3, but not SUMO-1. K-bZIP was found to contain a SIM at amino acid residues 72 to 76 , which was identical to that of the cellular SUMO-ligases, PIAS1, and PIASx. The sumoylation activity of K-bZIP was dependent on an intact SIM, and K-bZIP could catalyze its auto-sumoylation and the sumoylation of two K-bZIP-interacting proteins, p53 and RB. In contrast, Lefort et al. (2010) have reported that K-bZIP repression of interferon- $\alpha$ signaling was SIM-independent but was dependent on K-bZIP K158 sumoylation site, a Ubc9 consensus binding site. As described more detail below, K-Rta preferentially degrades SUMO-modified proteins, similar to an activity ascribed to HSV-1 ICP0 (Boutell et al., 2011). This suggests that a balance between sumoylation and SUMO-dependent degradation may be important for the KSHV life cycle. As the assembly and disassembly of Promyelocytic leukemia (PML; ND10) bodies at herpesvirus replication complexes are SUMO-dependent, modulation of the SUMO environment by K-bZIP and K-Rta during lytic replication cycle may help dictate whether viral replication will proceed or if latency will be established. Another potential role of K-bZIP may be to mark proteins that are destined to be targeted by the SUMO-targeting ubiquitin ligase (STUbL) activity of K-Rta (see below). Although not fully established, this idea is supported by the report that KSHV LANA2 increases PML sumoylation, which facilitates its degradation by a cellular ubiquitin ligase (Marcos-Villar et al., 2009).

\section{K-RTA}

$\mathrm{K}-\mathrm{Rta}$ is essential and sufficient to induce lytic reactivation of the latent KSHV genome in the BCBL-1 cell line model as well as de novo infection model (Lukac et al., 1998, 1999; Sun et al., 1998; Nakamura et al., 2003; Xu et al., 2005). K-Rta is a potent transcription factor, with an $\mathrm{N}$-terminal putative DNA binding domain and a C-terminal transactivation domain (Lukac et al., 1999; West and Wood, 2003). K-Rta possesses broad target sequence specificity, and various K-Rta responsive promoter elements have been identified (Lukac et al., 1999; Chang et al., 2002; Deng et al., 2002; Song et al., 2002; West and Wood, 2003). K-Rta has been reported to be phosphorylated (Lukac et al., 1999; Gwack et al., 2003), poly(ADP-ribosyl)ated (Gwack et al., 2003), and ubiquitylated (Yu et al., 2005). A schematic diagram of K-Rta modification sites is presented in Figure 2, and the consequences of these modifications will be discussed.

\section{K-Rta (ORF50)}

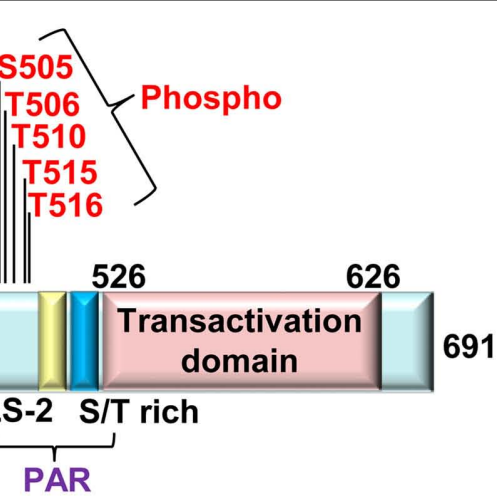

FIGURE 2 | Schematic representation of KSHV K-Rta. K-Rta protein and its post-translational modification sites as discussed in the text are depicted. Phosphorylation (Phospho) site numbering and PARylation (PAR) site localization of Gwack et al. (2003) is utilized. The K-Rta nuclear localization signals (NLS-1 and NLS-2), ring-finger like domain, SUMO interaction motifs (SIMs), and serine/threonine $(\mathrm{S} / \mathrm{T})$ rich regions are also indicated. Numbers indicate amino acid position. 
A

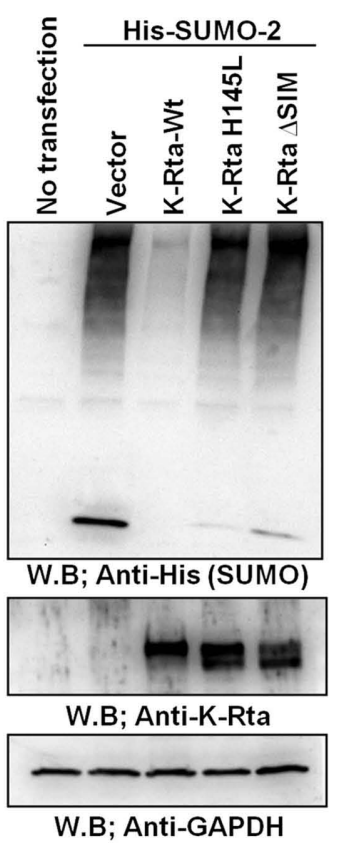

B

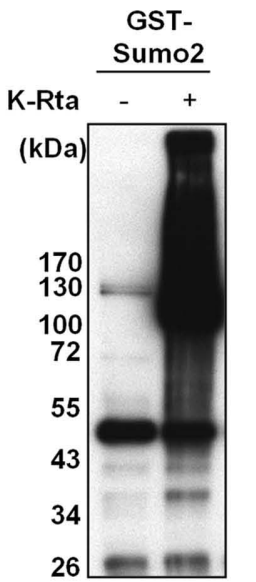

W.B: anti-Ubiquitin (HA)

C

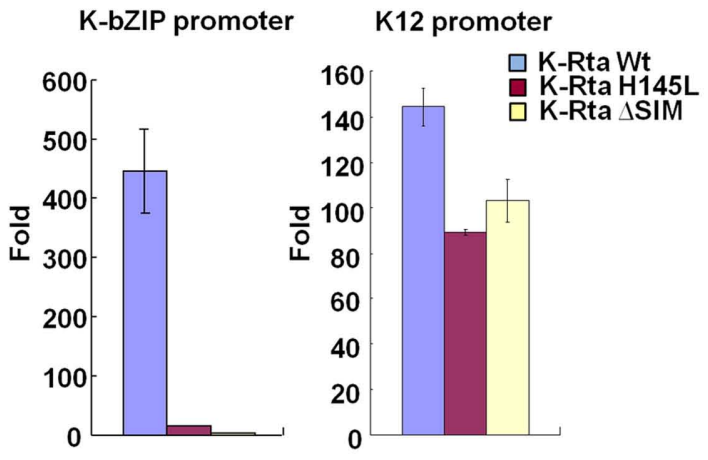

FIGURE 3 | SUMO-targeting ubiquitin ligase activity of KSHV K-Rta. (A) 293T cells were co-transfected with his-tagged SUMO-2 and the indicated K-Rta expression vector. Forty-eight hours later cellular lysates were prepared and analyzed by immunoblotting using the indicated antibodies. $(\mathrm{H} 145 \mathrm{~L}$, ring-finger like domain mutant; $\Delta$ SIM, SIM deletion mutant). (B) In vitro ubiquitin conjugation reactions were reconstituted using purified components (E1, Ube1, 25 nM; E2, Ubc H5a, 50 nM; E3, K-Rta, 150 nM). Reaction products were probed by immunoblotting. In the presence of K-Rta, GST-SUMO was conjugated with ubiquitin. (C) KSHV promoter reporter assay. 293T cells were co-transfected with K-Rta wt or mutant and the indicated KSHV promoter luciferase reporter. Forty eight hour later cellular lysates were prepared and assayed for luciferase activity. Values represent the mean fold induction (mean $\pm \mathrm{SD}, n=3$ determinations) relative to the value derived from reporter + empty vector set as $=1$ (K-Rta wt, blue; H145L, red; $\Delta$ SIM, yellow).

\section{PHOSPHORYLATION}

As part of an initial characterization of various aspects of ORF50 RNA and protein expression, structure, and function, K-Rta was found to be highly phosphorylated in mammalian cells (Lukac et al., 1999). By immunoblot analysis, K-Rta protein migrated with an apparent molecular mass of $\sim 110 \mathrm{kDa}$, greater than the predicted value of $74 \mathrm{kDa}$. Analysis of the K-Rta primary amino acid sequence revealed several potential phosphorylation sites including a C-terminal S/T-rich region, as well as additional consensus phosphorylation sites for the cellular serine/threonine kinases casein kinase-II and protein kinase C (PKC). Phosphatase treatment of K-Rta protein partially reduced the slower electrophoretic mobility of K-Rta suggesting that phosphorylation accounted for most of the anomalous migration observed during SDS-PAGE analysis of K-Rta. Subsequently, Gwack et al. (2003) identified the Ste20-like kinase hKFC and poly(ADP-ribose) polymerase 1 (PARP-1, see below) as proteins that specifically interact with the S/T-rich region of K-Rta. There are greater than 30 different Ste20-related kinases in humans and three members of a novel subfamily exhibit a high degree of homology to a Ste-like kinase originally isolated from chick cells (Yustein et al., 2000, 2003). hKFC is identical to JIK (Tassi et al., 1999), a kinase reported to be negatively regulated by upstream tyrosine kinase receptors. hKFC was shown to directly interact with K-Rta and phosphorylate at serine (S505) and threonine (T506, T510, T515, and T516) of K-Rta. Together with poly(ADP-ribosylation), phosphorylation inactivates K-Rta transcriptional activity and was hypothesized to serve as a contributor to the maintenance of KSHV latency. As hKFC has been shown to localize to the cytoplasm (Yustein et al., 2003), whereas K-Rta is primarily nuclear (Lukac et al., 1998), the interaction and phosphorylation by hKFC was proposed to potentially affect K-Rta localization.

\section{POLYADP-RIBOSYLATION (PARYLATION)}

Although the historical focus of PARP-1 has been its role in the cellular DNA damage response, studies have also revealed important roles for PARP-1 in transcriptional regulation. Poly-ADP-ribose (PAR) is a large negatively charged polymer that functions in both its free form or in the context of protein post-translational modifications. In mammalian cells, the majority of PAR production is catalyzed by PARP-1 and targets of PARP- 1 catalytic activity include histones, transcription factors, nuclear enzymes, and nuclear structural components (see Krishnakumar and Kraus, 2010; for a recent review). Similar to hKFC described above, PARP1 was identified by mass spectrometry as a protein that interacted with the S/T-rich region of K-Rta (Gwack et al., 2003). The interaction was mapped to the $\mathrm{NAD}^{+}$binding domain of PARP- 1 and this interaction mediated the PARylation of K-Rta. Synergistic effects between PARP-1 and hKFC on K-Rta binding and its subsequent modification were observed. The in vitro interaction between K-Rta and PARP-1 was increased by the addition of hKFC and conversely, in vitro interaction between K-Rta and hKFC was also 
increased with the addition of PARP-1. The reciprocal increases in binding were accompanied by enhanced phosphorylation and PARylation of K-Rta by hKFC and PARP-1, respectively. Moreover, PARP-1 and hKFC were found to act as repressors of K-Rta activity in reporter assays and in the efficiency of K-Rta-mediated viral reactivation. A combination of K-Rta mutational analysis, ChIP assays, and reporter activity measurements in PARP-1 knockout cells suggested a model in which PARP-1 and hKFC, acting as cellular sensors of herpesvirus infection, interfere directly or indirectly with the recruitment of K-Rta onto target promoters, leading to a decrease in K-Rta-mediated transcriptional activation (Gwack et al., 2003).

\section{SUMOYLATION}

Herpesvirus infections are controlled by acquired and innate defenses involving cellular, humoral, and cytokine-mediated responses. In recent years, a concept of intrinsic antiviral resistance has emerged as an additional antiviral defense mechanism that operates within individual cells. Unlike cytokine-mediated responses, intrinsic antiviral resistance involves the actions of preexisting cellular proteins that, in the case of herpesviruses, act to repress viral transcription. PML nuclear bodies (ND10 or PML$\mathrm{NBs)}$ have been identified as an important factor of intrinsic antiviral resistance against herpesvirus. Components of PML-NBs are recruited to sites associated with the viral chromosome soon after genomes enter the nucleus. Both SUMO-modification and SIMs of PML are required for formation of PML-NBs as well as recruitment to the viral genome. Viral gene expression is silenced by PML-NBs through deacetylation of local histones, which inhibits viral replication. Importantly, herpesvirus infection often disrupts PML-NBs, resulting in antagonism of both innate and intrinsic immune responses in early infection (Everett et al., 2006, 2008; Lukashchuk et al., 2006; Tavalai et al., 2006; Boutell et al., 2011).

Promyelocytic leukemia-NBs are mobile organelles that form distinct sub-compartments in the cell nucleus as described in a number of reviews (Bernardi and Pandolfi, 2007; Borden, 2008; Nagai et al., 2011). Assembly of PML-NBs, "storage" sites for SUMO, depends on the sumoylation of PML and other components such as Daxx, SP-100, and ATRX (Ishov et al., 1999; Zhong et al., 2000; Seeler et al., 2001; Fu et al., 2005). In addition, chromatin can be an integral part of PML-NBs. For example, a particular class of PML-NBs exists in tumor cells that maintain their telomeres in the absence of telomerase activity by a process referred to as alternative lengthening of telomeres (ALT). Recent studies identified that ALT is caused by the genomic mutations in Daxx or ATRX, both of which are localized in PML-NBs in a SUMO- and SIM-dependent manner (Lin et al., 2006; Berube et al., 2008; Heaphy et al., 2011). Interestingly, an ATRX-Daxx complex was recently identified as a histone chaperone, which specifically deposits the histone H3 variant, H3.3 (Drane et al., 2010; Goldberg et al., 2010; Lewis et al., 2010), leading to a possibility that PML-NBs function as a center of epigenetic gene regulation.

Like HSV-1 ICP0, our recent studies identified that K-Rta contained multiple SIMs and bound to SUMO in vitro. In co-transfection experiments with SUMO-2, K-Rta was found to markedly decrease the level of total cellular SUMO-2 modified proteins and mutations in the K-Rta SIM or ring-finger like domain significantly impaired this activity (Figure 3A). Using purified components, K-Rta was shown to conjugate ubiquitin to SUMO and SUMO-chains (Figure 3B). In addition, K-Rta preferentially targets SUMO-modified proteins for ubiquitylation; thus K-Rta is able to function as a STUbL which is capable of ubiquitylation of SUMO and SUMO conjugates in vitro and in vivo (Figures 3A,B; Izumiya et al., unpublished). The prototypical STUbL, cellular RNF4, was first identified as a ubiquitin ligase that functions to maintain genomic stability, and was recently reported to be a ubiquitin ligase targeting SUMO-containing proteins, including sumoylated PML (Sun et al., 2007; Tatham et al., 2008). Members of this ubiquitin ligase family include mammalian RNF4 and the heterodimers Hex3-Slx8 and Rfp1-Slx8 in S. cerevisiae and S. pombe, respectively (Uzunova et al., 2007; Xie et al., 2007). Similar to HSV-1 ICP0 and cellular RNF4, KSHV K-Rta disrupts the PML body in ubiquitin ligase dependent fashion (data not shown). Sumoylation is often linked to gene repression due to its ability to serve as a platform to recruit strong co-repressors such as SETDB1, HDAC1, and HDAC2 via SUMO-SIM interactions. Accordingly, K-Rta's ability to remove SUMO is relevant to its transactivation functions. Mutations in SIM or ring-finger like domain significantly impaired K-Rta transactivation activity. We observed that the K-bZIP promoter, a well recognized promoter transactivated by K-Rta, is activated by wild-type K-Rta, but not by H145L (ring-finger) mutant or a K-Rta SIM mutant (Figure 3C). Interestingly, the K12 (Figure 3C) or PAN promoter (not shown) were relatively unaffected. Thus, it is likely that there are SUMO-dependent and SUMO-independent promoters, which may depend on local chromatin structure and mode of K-Rta recruitment. These results suggest that K-Rta, in addition to being a strong transactivator, has an ability to regulate the local SUMO environment; the latter may contribute to its former functions through disassembly of SIM-containing co-repressors.

\section{UBIQUITYLATION}

K-Rta was found to contain an E3 ubiquitin ligase activity which was associated with the regulation of the cellular levels of interferon regulatory factor 7 (IRF7; Yu et al., 2005). During the characterization of this activity, it was determined that that K-Rta was subjected to self-ubiquitylation. Three residues were identified in the Cys/His-rich region of K-Rta which was found to be critical for both K-Rta auto-ubiquitylation and for degradation of IRF7. These residues, Cys131, Cys141, and His145 were proposed to be part of a novel Cys-rich domain extending from K-Rta amino acids 118-207 (C3HC3). However, this ring-finger like domain shows no significant homology with any previously described sequences conserved among E3 ligases. Based on analogy to other transcriptional activators, the K-Rta auto-ubiquitylation function may serve as a "degron" to activate gene expression (Muratani and Tansey, 2003; Kodadek et al., 2006). Indeed, K-Rta mutants that harbor a mutation in ring-finger like domain are more stable than K-Rta Wt. This stability is associated with a concomitant loss of transactivation function (Figure 3C). Further studies are required to dissect the contribution of K-Rta auto-ubiquitylation and repressor degradation activity to K-Rta transactivation (Yang et al., 2008). 


\section{LANA}

KSHV (LANA/ORF73) is key regulatory protein that is essential for the establishment and maintenance of viral latency. LANA is a DNA binding protein that is a functional homolog of EBV EBNA1. LANA binds to the viral latent origin of replication, located at the terminal repeat (TR) sequence of the KSHV genome, and is highly expressed in all KSHV-associated disorders (Kedes et al., 1997; Schulz, 2001; Hu et al., 2002; Komatsu et al., 2004; Verma et al., 2007; Kelley-Clarke et al., 2009; Ganem, 2010; Mesri et al., 2010). LANA functions as both a transcriptional activator and a repressor depending on the context of promoters and cell line interrogated (An et al., 2005; Kelley-Clarke et al., 2009). Accordingly, LANA has been shown to associate with a broad range of transcriptional regulators such as RBP-Jк, CBP, Daxx, BRD2, RB, p53, and Sp-1 (Friborg et al., 1999; Platt et al., 1999; Radkov et al., 2000; Lim et al., 2001; Verma et al., 2004; Lan et al., 2005; Murakami et al., 2006). LANA function is regulated and/or mediated by protein partner interaction. In addition, LANA activity is regulated by post-translational modifications, including phosphorylation (Platt et al., 1999; Fujimuro et al., 2005; Bajaj et al., 2006; Varjosalo et al., 2008; Cheng et al., 2009; Cha et al., 2010), acetylation (Lu et al., 2006), PARylation (Ohsaki et al., 2004), sumoylation (Izumiya et al., unpublished), and arginine methylation (Campbell et al., 2011). These modification sites are depicted in Figure 4.

\section{PHOSPHORYLATION}

Latency-associated nuclear antigen interacts with and is phosphorylated by several kinases including glycogen synthase kinase 3 (GSK-3; Fujimuro and Hayward, 2003; Fujimuro et al., 2005; Liu et al., 2007), Pim-1 and Pim-3 (Bajaj et al., 2006; Varjosalo et al., 2008; Cheng et al., 2009), and DNA-PK/Ku (Cha et al., 2010). LANA is also phosphorylated by an unidentified kinase mediated through an interaction of LANA with RING3 (Platt et al., 1999). In studies designed to characterize the interaction of LANA with RING3, Platt et al. (1999) first described phosphorylation of LANA in vitro. The interaction between RING3 and LANA resulted in serine/threonine phosphorylation of LANA on residues located between LANA amino acids 951 and 1107, and binding of
RING3 to LANA facilitated the phosphorylation of LANA via an unknown co-immunoprecipitating kinase. However, the identity of the kinase and its role in the KSHV life cycle remains to be determined.

Using yeast two-hybrid screening with LANA as the bait protein, GSK-3 was shown to interact with LANA and through coimmunoprecipitation LANA interacted with both GSK-3 $\alpha$ and GSK- $3 \beta$ in transfected cells. This interaction resulted in increased nuclear accumulation of GSK-3 $\beta$ (Fujimuro and Hayward, 2003). Fujimuro et al. (2005) further showed that GSK-3 $\beta$ phosphorylated multiple $\mathrm{N}$-terminal residues of LANA in vitro and in KSHV infected PEL cells. Phosphorylation of LANA by GSK-3 $\beta$ was necessary for the LANA/GSK- $3 \beta$ interaction. GSK-3 $\beta$ predominantly phosphorylates pre-phosphorylated (primed) substrates that contain the motif $\mathrm{S} / \mathrm{TxxxS} / \mathrm{Tp}$, where the +4 position $\mathrm{S}$ or $\mathrm{T}$ residue has prior phosphorylation mediated by another priming kinase (reviewed in Forde and Dale, 2007). LANA was found to require priming phosphorylation in vitro in order to serve as a GSK-3 $\beta$ substrate; mitogen-activated protein kinase (MAPK) and casein kinase-I (CK-I) could function as priming kinases, while CK-II could not. Subsequently ERK $1 / 2$ was also found to phosphorylate and prime LANA for subsequent GSK-3 $\beta$ phosphorylation (Liu et al., 2007). A GSK-3 $\beta$ priming pocket mutant (R96A) could not bind to LANA. Interestingly, in co-transfection experiments, GSK$3 \beta$ present in a LANA-GSK-3 $\beta$ complex was unable to phosphorylate a primed peptide substrate. These results were interpreted to suggest that GSK- $3 \beta$ phosphorylation sites on LANA function to increase the affinity of LANA binding to GSK- $3 \beta$ and simultaneously provide a competitive substrate that minimizes potential phosphorylation events on other non-complexed substrates (Fujimuro et al., 2005). Taken together, these results suggested a model in which LANA sequesters GSK-3 $\beta$ in the nucleus leading to dysregulation of the kinase function in both the cytoplasmic ( $\beta$-catenin stability) and nuclear (gene reprogramming) compartments (Fujimuro and Hayward, 2003; Fujimuro et al., 2005; Liu et al., 2007). Aspects of this model have been challenged by a report, in which LANA overexpression showed no effect on endogenous $\beta$-catenin levels (Hagen, 2009); this report

\section{LANA (ORF73)}

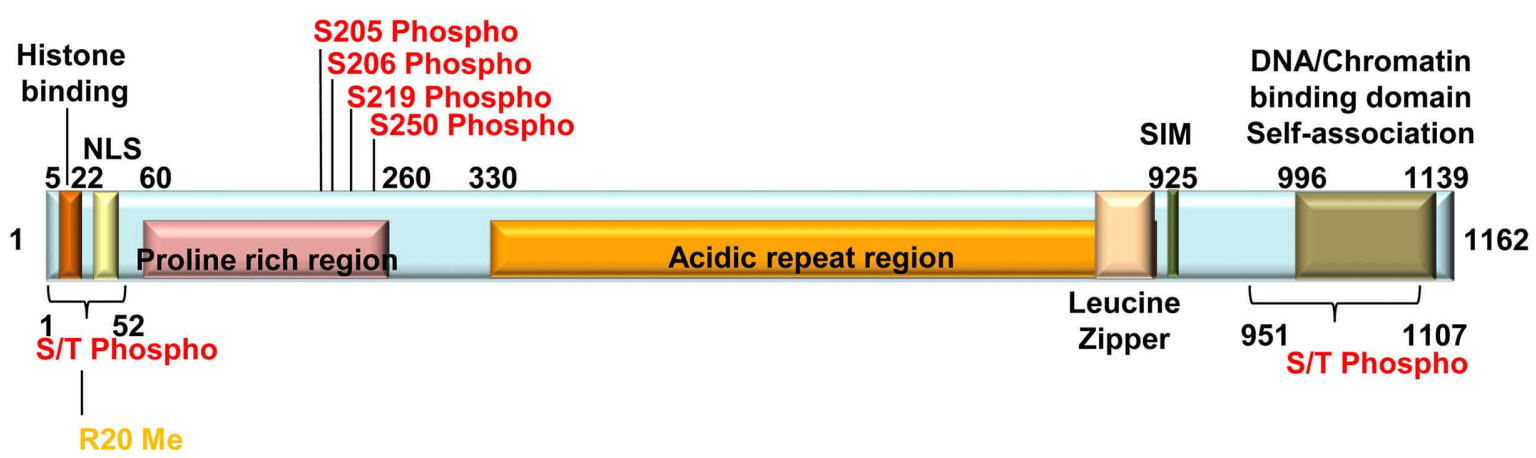

FIGURE 4 | Schematic representation of KSHV LANA. LANA protein and its post-translational modification sites as discussed in the text are depicted. Phosphorylation (Phospho) sites and the arginine methylation (Me) site are shown. The $\mathrm{N}$-terminal histone binding domain, nuclear localization signal (NLS) and SUMO interaction motif (SIM) are also indicated. Numbers indicate amino acid position. 
demonstrates the complexity of the interaction and its context dependence.

Bajaj et al. (2006) identified LANA as a substrate for Pim-1 kinase. The Pim-1 gene was first identified as a common proviral insertion site of the Moloney murine leukemia virus (proviral insertion site MuLV; Cuypers et al., 1984). Pim-1 encodes a Ser/Thr kinase, which belongs to the group of calcium/calmodulinregulated kinases. This gene is expressed primarily in B-lymphoid and myeloid cell lines, and is overexpressed in hematopoietic malignancies. Pim-1 plays a role in signal transduction in blood cells, contributing to cell growth and survival, and thus may provide a selective advantage in tumorigenesis (Bachmann and Moroy, 2005). Pim-1 was shown to bind to the C-terminus of LANA and to phosphorylate LANA at its N-terminus, specifically serine residues 205 and 206. Using human kinome expression screening, Varjosalo et al. (2008) confirmed that Pim-1 could phosphorylate LANA and augment viral reactivation primed by low levels of K-Rta. Cheng et al. (2009) further showed that another Pim family kinase member, namely Pim-3, could also phosphorylate LANA at serines 205 and 206. Moreover, ectopic expression of Pim-1 or Pim-3 in rKSHV.219-infected Vero cells enhanced the level of reactivation when co-expressed with low levels of exogenous K-Rta. Mechanistically, Pim-1 and Pim-3 phosphorylation of LANA was shown to antagonize LANA-dependent transcriptional repression of a synthetic TR reporter as well as decrease the ability of LANA to inhibit KRta auto-activation of its own promoter. As Pim-1 and Pim-3 are up-regulated during viral reactivation (Cheng et al., 2009) a model was proposed in which these kinases participate in reactivation by negative modulation of LANA function via direct phosphorylation.

Latency-associated nuclear antigen-dependent latent episomal replication is also regulated by phosphorylation. The DNA-PK/Ku complex bound and phosphorylated LANA (Cha et al., 2010). Uninfected B cell extracts were fractionated over a GST affinity resin containing LANA amino acids $1-52$. Among the proteins found to interact with this LANA fragment were DNAdependent protein kinase (DNA-PK) catalytic subunit (DNAPKcs), Ku70, and Ku86. In agreement with Ohsaki et al., 2004; see below), Cha et al. also identified PARP-1 as a LANA interacting protein. The phosphorylation of LANA by the DNA-PK/Ku complex was wortmannin-sensitive and mapping data suggested that LANA was phosphorylated at multiple positions. Interestingly, transient replication of a TR-containing plasmid was reduced when a phosphorylation-competent N-terminal LANA fragment was fused to a C-terminal LANA fragment that supported TR-dependent replication. Two similarly designed mutants that were not phosphorylated by DNA-PK/Ku were not defective in this assay. Overexpression of Ku70 also impaired transient TRdependent replication in co-transfection assays conducted in 293T cells. Although overexpression of Ku86 did not affect transient replication assays set up in $293 \mathrm{~T}$ cells, the authors noted an increase in TR-dependent replication when the assays were conducted in Ku86 heterozygous HCT116 Ku86+/- cells when compared to a HCT116 Ku86+/+ background. Taken together, these results suggest that the DNA-PK/Ku complex negatively regulates latent episomal replication.

\section{ACETYLATION}

Latency-associated nuclear antigen was reported to be lysine acetylated in response to sodium butyrate or trichostatin A-mediated reactivation (Lu et al., 2006). Although the site(s) of lysine acetylation on LANA were not mapped, LANA acetylation was observed in the presence of HDAC inhibition using either ectopic LANA expression or endogenous LANA in BCBL-1 cells as substrate. LANA acetylation was observed in HDAC inhibitor-treated cells using immunological detection with antibodies specific for acetyl lysine or by metabolic labeling of cells with $\left[{ }^{14} \mathrm{C}\right]$-sodium acetate followed by fluorography. LANA acetylation occurred during reactivation and this was accompanied by disruption of the interaction between LANA and the cellular proteins Sp-1 and histone $\mathrm{H} 2 \mathrm{~B}$. ChIP analysis of HDAC inhibitor-treated BCBL- 1 cells also revealed a loss of LANA from the K-Rta promoter region that occurred within the same time frame as LANA acetylation. Together, these results suggest that LANA acetylation contributes to the ORF50 transcriptional de-repression during the early stage of KSHV lytic cycle reactivation.

\section{PARYLATION}

As mentioned previously, PARP has been historically studied in the context of the DNA damage response, but PARP-1 has more recently been linked to the regulation of transcription and chromatin structure. PARP-1 binds to a variety of DNA structures, nucleosomes, and chromatin-associated proteins (Krishnakumar and Kraus, 2010). Ohsaki et al. (2004) reported that LANA was a target of PARP-1 and was poly(ADP-ribosyl)ated. Using a TR affinity column and BC3 cell extracts, PARP-1 was isolated as a TR interacting protein. TR binding by PARP-1 was independent of LANA and was also observed with BJAB cell extracts; however the affinity column elution profiles of PARP-1 and LANA were similar. In addition, PARP-1 was found to co-localize with LANA in the nucleus, suggesting that LANA may be a potential PARP-1 substrate. A small fraction of LANA present in BC3 anti-LANA immunoprecipitates was found to react with anti-PAR antibody. Moreover, when LANA immunoprecipitates were incubated with purified human PARP-1 and NAD in vitro, a species consistent with NAD-incorporated LANA was observed. Intriguingly, PARP-1 activity appeared to control viral copy number in infected cells. When BC3 cells were treated with hydroxyurea, a compound that elevates PARP activity, a $\sim 4$-fold decrease in KSHV copy number was observed over the course of a 5-day treatment period. Conversely, when cells were similarly treated with compounds that decreased PARP activity (niacinamide and 3 -aminobenzamide), KSHV copy numbers increased $\sim 8$-fold. Interestingly, for hydroxyurea-treated cells, the decrease in viral copy number was accompanied by an increase in the PARylation signal detected in LANA immunoprecipitates. Although mechanistic details remain to be elucidated, these results suggest that the viral replication and/or episome partitioning functions of LANA may be influenced by cellular PARylation activity. Similar results were reported for EBV EBNA1, which was found interact with both PARP-1 (Deng et al., 2002; Tempera et al., 2010) and telomere-associated PARPs, Tankyrases- 1 and -2 (Deng et al., 2002, 2005). Both PARP-1 and Tankyrase- 1 were found to catalyze PARylation of EBNA1 and negatively regulate EBNA1 origin of 


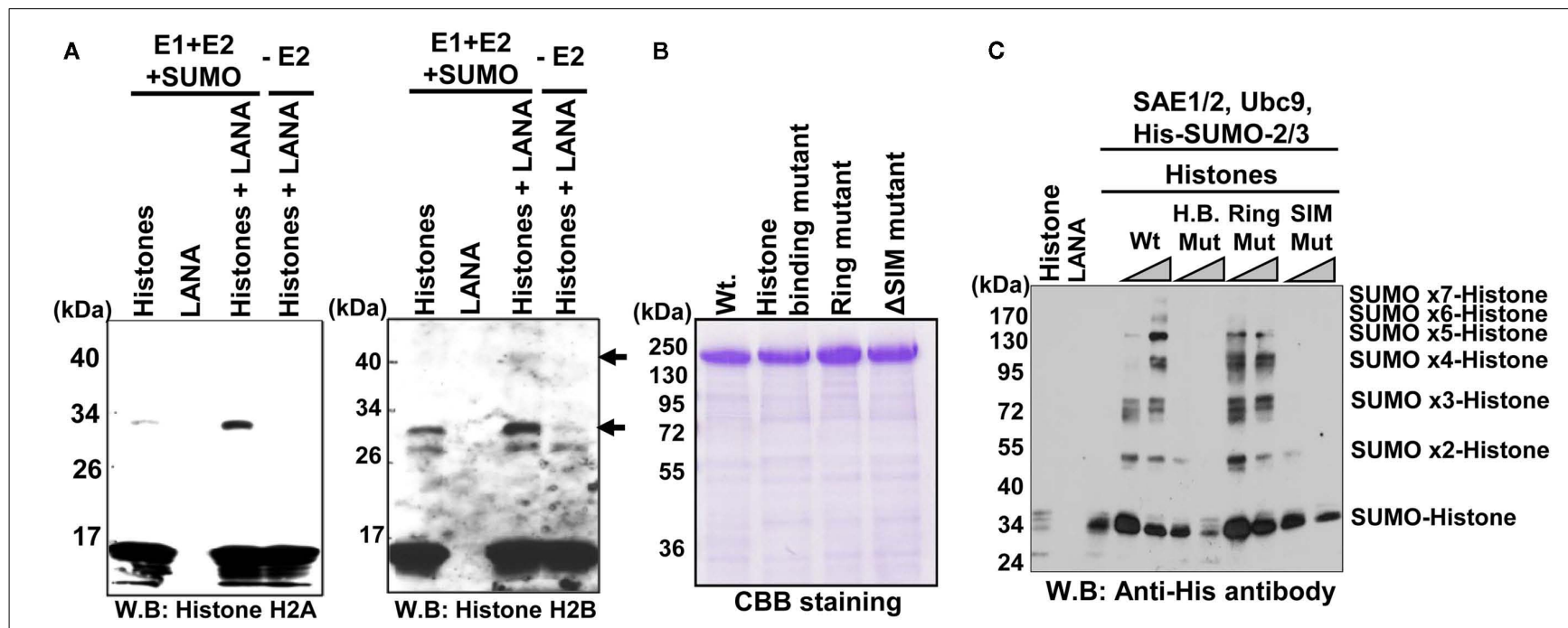

D

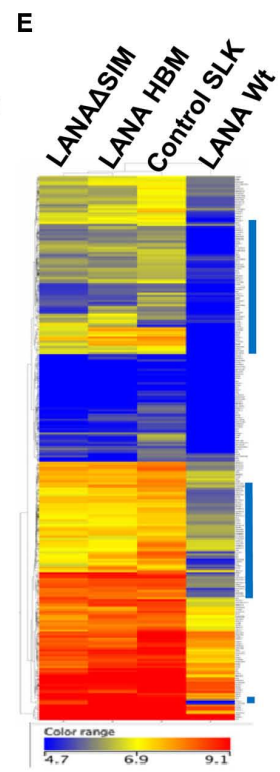

FIGURE 5 | Latency-associated nuclear antigen enhances histone sumoylation in vitro and in vivo. (A) LANA enhances histone $\mathrm{H} 2 \mathrm{~A}$ and H2B SUMO-modification in vitro. The in vitro SUMO conjugation reaction was performed with purified histone octamer ( $5 \mu \mathrm{g}$; Roche) as a substrate. SAE1/2 (E1, $50 \mathrm{nM})$, Ubc9 (E2, $50 \mathrm{nM})$, and HA-SUMO-2 $(50 \mu \mathrm{M})$ were incubated in reaction buffer containing $1 \mathrm{mM}$ ATP and $\mathrm{MgCl}_{2}$ with or without a catalytic amount of LANA $(50 \mathrm{nM})$. In the presence of LANA, H2A, and H2B SUMO-modification is enhanced. The arrows indicate SUMO-modified histones, which were increased in SUMO-reaction mixtures supplemented with LANA (three lanes from left). Full-length LANA Wt and mutant proteins were prepared with recombinant baculoviruses (B) and used as an E3 SUMO-ligase for in vitro SUMO conjugation reactions. CBB, Coomassie brilliant blue. (C) Histone binding domain and SUMO-interacting motif of LANA are necessary for its SUMO-ligase function. LANA Wt but neither a histone binding mutant (HB Mut) or SUMO-binding mutant (SIM Mut) can catalyze SUMO-modification of histones. The corresponding number of SUMO-chains is indicated on the right $(20 \mathrm{kDa}$ increments). LANA ring-finger like domain is dispensable for LANA-mediated SUMO conjugation of histones (Ring Mut). (D) Correlation between LANA recruitment sites and SUMO-enriched loci on the latent KSHV genome. ChIP analyses were performed with anti-LANA or anti-SUMO-2 antibodies using latent BCBL-1 cells. ChIP with LANA antibody was hybridized with custom-made KSHV tiling arrays and SUMO-ChIP was directly analyzed by sequencing (ChIP-seq). Enrichment over input DNA is shown (upper panel). Counts and position of sequence reads of SUMO-ChIP are shown in the bottom panel. SUMO-2 is enriched where LANA is recruited. Annotation of the KSHV genome is shown in the middle panel. The KSHV terminal repeat region is located at the extreme right of the annotated genome. (E) cDNA microarray analyses. RNA was prepared from indicated LANA stable cells or vector control stable cells and hybridized to Affymetrix human whole genome U133 plus 2.0 arrays. Raw data was subjected to baseline transformation and analyzed by hierarchical clustering using GeneSpring GX11 software. Genes highly repressed by LANA wt but not mutants are indicated by blue vertical lines to the right. plasmid replication (OriP) activity in a PAR-dependent manner. Although the effects of the cellular DNA damage response on viral replication and gene expression are multi-faceted and enigmatic (Lilley et al., 2007; Weitzman et al., 2010) these result suggest that 
current PARP-1 inhibitors (see Rouleau et al., 2010; for a recent review) under evaluation for treatment of certain malignancies might be worth examining for their efficacy as a treatment for KSHV infected individuals.

\section{ARGININE METHYLATION}

Our lab has determined that LANA is a substrate for protein arginine methyltransferase (PRMT)-1 in vitro and in vivo, and the major methylation site was mapped to the N-terminus of LANA (Arg 20; Campbell et al., 2011). Although, there was no effect of methylation on LANA nuclear localization or nuclear matrix association, methylation at this position did affect the interaction of LANA with histone octamers in vitro and with KSHV chromatin in vivo. In vitro, the histone octamer-LANA interaction was strengthened when the R20 site was replaced with a methyl-mimic residue, phenylalanine (R20F). Similarly, using LANA ChIP analysis, consistently higher amounts of KSHV DNA could be recovered from cells harboring latent KSHV bacmids containing the LANA R20F mutant. These results suggest that arginine methylation at position R20 may potentially influence a sub-population of LANA protein within infected cells resulting in increased interaction of LANA with KSHV-associated chromatin.

\section{SUMOYLATION}

Sumoylation-modification plays important roles in assembling protein complexes to regulate gene expression. Recent studies support a model in which the SUMO-modification has a significant impact on heterochromatin formation by recruiting SIMcontaining co-repressors (Uchimura et al., 2006; Stielow et al.,

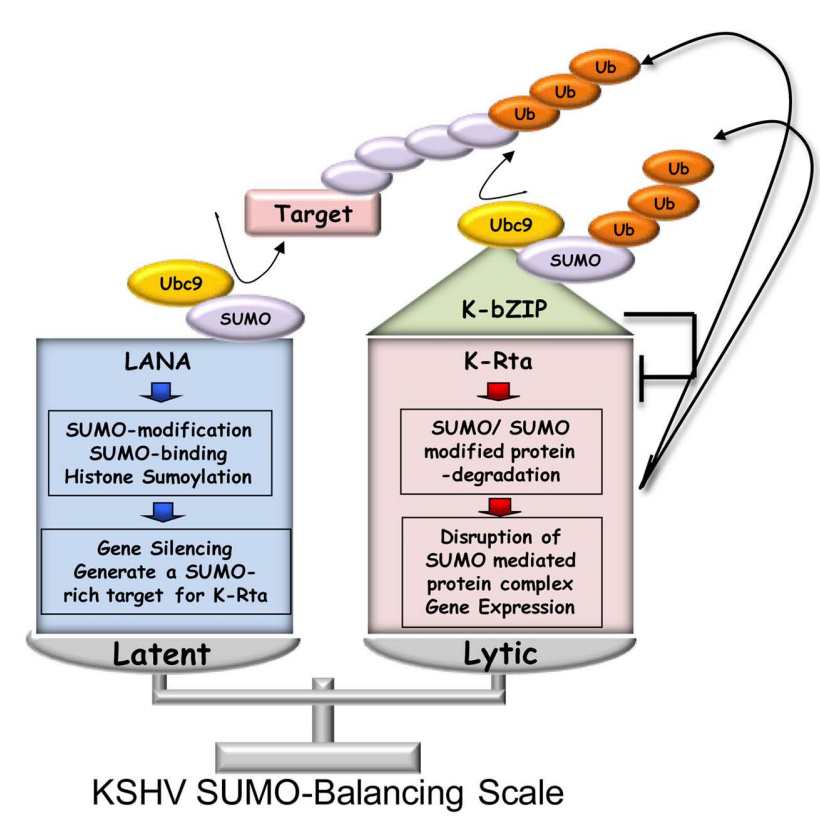

FIGURE 6 | KSHV SUMO-dependent balance. The outcome of the KSHV life cycle is depicted as a balance between the functions of the viral factors LANA, K-Rta, and K-bZIP which are mediated through their

SUMO-dependent activities. The SUMO E3 ligase activities of K-bZIP and LANA and the STUbL activity of K-Rta are shown (see text for details).
2008). Accordingly, our lab has found that LANA is both heavily sumoylated and binds SUMO. By creating a SUMO-rich environment on local chromatin, we propose LANA may be able to condense chromatin, which leads to gene silencing mediated through its SIM domain. Interestingly, when we searched the LANA protein sequence for the SUMO-modification consensus motif $(\mathrm{V} / \mathrm{I} / \mathrm{L}-\mathrm{K}-\mathrm{x}-\mathrm{E})$, which is a well-established Ubc9 recognition sequence, there was no such motif. This observation indicates that LANA itself may bind SUMO to recruit Ubc9. Since LANA is heavily modified by SUMO in the absence of an E3 SUMOligase in vitro, LANA is likely to bind to the SUMO-Ubc9 complex ("active-SUMO") directly for its own SUMO-modification. Further detailed SUMO-binding analyses with a panel of GSTLANA deletion proteins confirmed that LANA possesses a SIM domain at its C-terminus. These result prompted us to investigate if LANA functions as an E3 SUMO-ligase, because the recruitment of SUMO-Ubc9 complex (active-SUMO) is one of the mechanisms for catalyzing the conjugation of SUMO. Although LANA associates with multiple proteins, we decided to focus on chromatin components and histone modifying enzymes involved in epigenetic gene regulation. We chose to use histone octamers as substrates, because LANA has been shown to associate with histones at the H2A-H2B acidic patch (Barbera et al., 2006). In addition, SUMO-modification of histones, especially histone $\mathrm{H} 2 \mathrm{~B}$, is linked to chromatin condensation (Nathan et al., 2006). Our results confirmed that LANA can enhance SUMO-modification of histones in vitro (Figure 5A). The purified proteins that were used in the in vitro SUMO-reactions are shown in Figure 5B. Sumoylation reactions using two LANA mutants clearly show that both a histone binding mutation and SIM mutation significantly diminished histone targeting SUMO-ligase activity, supporting the idea that LANA needs to bind both substrate and SUMO for the activity (Figure 5C). Our results demonstrate for the first time that a viral protein acts as an E3 SUMO-ligase to increase SUMOmodification of histones, and which is likely to be involved in the formation of localized SUMO-rich chromatin. Next, we examined the distribution of SUMO and LANA across the latent KSHV episome. Comparison of the SUMO-ChIP assay with KSHV tiling array data indicated that the overlap of local SUMO occupancy with LANA binding sites within the KSHV genome was extensive (Figure 5D). Together, these experiments show that LANA can increase the content of histone SUMO-modification in vitro (isolated system without other proteins), and in vivo at the local chromatin level. Based on these data, we propose that LANA increases local SUMO-modification, and thus impacts and initiates epigenetic changes at these sites. These epigenetic changes may causally associate with the observed silencing of both the KSHV genome and at specific genomic loci of the host cell. We therefore examined whether assembly of a SUMO-mediated complex would translate into gene repression. If LANA SUMO-ligase function is important for such a complex formation, the LANA SUMO-ligase mutants [SUMO-binding mutant $(\triangle \mathrm{SIM})$ and histone binding mutant $(\mathrm{HBM})]$ should have impaired gene repression function. To answer this question, cDNA microarray analyses were employed to probe the significance of histone targeting SUMOligase function in gene regulation. LANA wild-type and $\triangle$ SIM and HBM mutant stable SLK cells were generated. These cells 
lines expressed equivalent amounts of LANA or mutant LANA proteins when examined by western blotting with anti-Flag antibody. As shown in Figure 5E, both $\triangle$ SIM and HBM mutants had significantly impaired gene repression activity relative to wild-type LANA cells; these two cell lines showed gene expression patterns very similar to vector control SLK cells. Taken together, these results demonstrate that LANA recruits SUMO-Ubc9 complexes through SUMO-binding, and facilitates local histone SUMOmodification. Importantly, enrichment of SUMO at LANA binding sites may also prepare the KSHV genome for reactivation by generating a suitable target for K-Rta. Obviously, further studies are required to test this possibility. A putative SUMO-mediated KSHV latency-reactivation model is depicted in Figure 6.

\section{SUMMARY}

Although there are numerous studies describing post-translational modifications of several KSHV regulatory factors, there appears to be a fundamental gap in our knowledge as to precisely how, where

\section{REFERENCES}

Adamson, A. L., and Kenney, S. (2001). Epstein-Barr virus immediate-early protein BZLF1 is SUMO-1 modified and disrupts promyelocytic leukemia bodies. J. Virol. 75, 2388-2399.

An, F. Q., Compitello, N., Horwitz, E., Sramkoski, M., Knudsen, E. S., and Renne, R. (2005). The latency-associated nuclear antigen of Kaposi's sarcoma-associated herpesvirus modulates cellular gene expression and protects lymphoid cells from p16 INK4A-induced cell cycle arrest. J. Biol. Chem. 280, 3862-3874.

Bachmann, M., and Moroy, T. (2005). The serine/threonine kinase Pim1. Int. J. Biochem. Cell Biol. 37, 726-730.

Bajaj, B. G., Verma, S. C., Lan, K., Cotter, M. A., Woodman, Z. L., and Robertson, E. S. (2006). KSHV encoded LANA upregulates Pim-1 and is a substrate for its kinase activity. Virology 351, 18-28.

Barbera, A. J., Chodaparambil, J. V., Kelley-Clarke, B., Joukov, V., Walter, J. C., Luger, K., and Kaye, K. M. (2006). The nucleosomal surface as a docking station for Kaposi's sarcoma herpesvirus LANA. Science 311, 856-861.

Bernardi, R., and Pandolfi, P. P. (2007). Structure, dynamics and functions of promyelocytic leukaemia nuclear bodies. Nat. Rev. Mol. Cell Biol. 8, 1006-1016.

Berube, N. G., Healy, J., Medina, C. F., Wu, S., Hodgson, T., Jagla, M., and Picketts, D. J. (2008). Patient mutations alter ATRX targeting to PML nuclear bodies. Eur. J. Hum. Genet. 16, 192-201.

Boggio, R., Passafaro, A., and Chiocca, S. (2007). Targeting SUMO E1 to ubiquitin ligases: a viral strategy to counteract sumoylation. J. Biol. Chem. 282, 15376-15382.

Borden, K. L. (2008). Pondering the puzzle of PML (promyelocytic leukemia) nuclear bodies: can we fit the pieces together using an RNA regulon? Biochim. Biophys. Acta 1783, 2145-2154.

Bossis, G., Malnou, C. E., Farras, R., Andermarcher, E., Hipskind, R., Rodriguez, M., Schmidt, D., Muller, S., Jariel-Encontre, I., and Piechaczyk, M. (2005). Downregulation of c-Fos/c-Jun AP-1 dimer activity by sumoylation. Mol. Cell. Biol. 25, 6964-6979.

Boutell, C., Cuchet-Lourenco, D., Vanni, E., Orr, A., Glass, M., McFarlane, S., and Everett, R. D. (2011). A viral ubiquitin ligase has substrate preferential SUMO targeted ubiquitin ligase activity that counteracts intrinsic antiviral defence. PLoS Pathog. 7, e1002245. doi:10.1371/journal.ppat.1002245

Campbell, M., Chang, P.-C., Huerta, S., Izumiya, C., Davis, R., Tepper, C. G., Kim, K. Y., Shevchenko, B., Wang, D.-H., Jung, J. U., Luciw, P. A., Kung, H.-J., and Izumiya, Y. (2011). Protein arginine methyltransferase 1-directed methylation of Kaposi sarcoma-associated herpesvirus latency-associated nuclear antigen. J. Biol. Chem. doi: 10.1074/jbcM111. 289496

Cha, S., Lim, C., Lee, J. Y., Song, Y.-J., Park, J., Choe, J., and Seo, T. (2010). DNA-PK/Ku complex binds to latency-associated nuclear antigen and negatively regulates Kaposi's sarcoma-associated herpesvirus latent replication. Biochem. Biophys. Res. Commun. 394, 934-939.

and when these modifications affect the regulatory functions associated with each protein. These are very difficult questions to address for several reasons. The modifications affect only a small percentage of the total pool of a particular molecule, are transient in nature, and, in many cases, detection is technically demanding. Nevertheless, this area of herpesvirus research should be fruitful in yielding novel insights into both virus-host interactions and basic cellular signaling pathways. The recent advancement of proteomic approaches will certainly help to answer these questions.

\section{ACKNOWLEDGMENTS}

We would like to thank our collaborators Pei-Ching Chang, Ryan Davis, and Clifford G. Tepper for their technical assistance. We also would like to thank Dr. Hsing-Jien Kung for his generous support. We apologize to those authors whose work was not cited due to space limitations. This work was supported by grants from the National Institutes of Health (R01-CA147791 and P01-DE19085 to Yoshihiro Izumiya).

Chang, P. C., Fitzgerald, L. D., van Geelan, A., Izumiya, Y., Ellison, T. J., Wang, D.-H., Ann, D. K., Luciw, P. A., and Kung, H. J. (2009). Kruppelassociated box domain-associated protein-1 as a latency regulator for Kaposi's sarcoma-associated herpesvirus and its modulation by the viral protein kinase. Cancer Res. 69, 5681-5689.

Chang, P. C., Izumiya, Y., Wu, C. Y., Fitzgerald, L. D., Campbell, M., Ellison, T. J., Lam, K. S., Luciw, P. A., and Kung, H. J. (2010). Kaposi's sarcoma-associated herpesvirus (KSHV) encodes a SUMO E3 ligase that is SIM-dependent and SUMO-2/3-specific. J. Biol. Chem. 285, 5266-5273.

Chang, P. J., Shedd, D., Gradoville, L., Cho, M. S., Chen, L. W., Chang, J., and Miller, G. (2002). Open reading frame 50 protein of Kaposi's sarcoma-associated herpesvirus directly activates the viral PAN and K12 genes by binding to related response elements. J. Virol. 76, 3168-3178.

Cheng, F., Weidner-Glunde, M., Varjosalo, M., Rainio, E. M., Lehtonen, A., Schulz, T. F., Koskinen, P. J., Taipale, J., and Ojala, P. M. (2009). KSHV reactivation from latency requires $\mathrm{Pim}-1$ and Pim-3 kinases to inactivate the latency-associated nuclear antigen LANA. PLoS Pathog. 5, e1000324. doi:10.1371/journal.ppat.1000324

Colombo, R., Boggio, R., Seiser, C., Draetta, G. F., and Chiocca, S. (2002). The adenovirus protein Gaml interferes with sumoylation of histone deacetylase 1. EMBO Rep. 3, 1062-1068.

Cuypers, H. T., Selten, G., Quint, W., Zijlstra, M., Maandag, E. R., Boelens, W., van Wezenbeek, P.,
Melief, C., and Berns, A. (1984) Murine leukemia virus-induced Tcell lymphogenesis: integration of proviruses in a distinct chromosomal region. Cell 37, 141-150.

Daniel, A. R., Faivre, E. J., and Lange, C. A. (2007). Phosphorylationdependent antagonism of sumoylation derepresses progesterone receptor action in breast cancer cells. Mol. Endocrinol. 21, 2890-2906.

Daniel, A. R., and Lange, C. A. (2009). Protein kinases mediate ligand-independent derepression of sumoylated progesterone receptors in breast cancer cells. Proc. Natl. Acad. Sci. U.S.A. 106, 14287-14292.

Deng, H., Song, M. J., Chu, J. T., and Sun, R. (2002). Transcriptional regulation of the interleukin- 6 gene of human herpesvirus 8 (Kaposi's sarcoma-associated herpesvirus). $J$. Virol. 76, 8252-8264.

Deng, Z., Atanasiu, C., Zhao, K., Marmorstein, R., Sbodio, J. I., Chi, N. W. and Lieberman, P. M. (2005). Inhibition of Epstein-Barr virus OriP function by tankyrase, a telomereassociated poly-ADP ribose polymerase that binds and modifies EBNA1. J. Virol. 79, 4640-4650.

Drane, P., Ouararhni, K., Depaux, A., Shuaib, M., and Hamiche, A. (2010). The death-associated protein DAXX is a novel histone chaperone involved in the replicationindependent deposition of $\mathrm{H} 3.3$. Genes Dev. 24, 1253-1265.

Ellison, T. J., Izumiya, Y., Izumiya, C., Luciw, P. A., and Kung, H. J. (2009). A comprehensive analysis of recruitment and transactivation potential of K-Rta and K-bZIP during reactivation of Kaposi's sarcomaassociated herpesvirus. Virology 387 , 76-88. 
Everett, R. D., Parada, C., Gripon, P., Sirma, H., and Orr, A. (2008). replication of ICP0-null mutant herpes simplex virus type 1 is restricted by both PML and sp100. J. Virol. 82, 2661-2672.

Everett, R. D., Rechter, S., Papior, P., Tavalai, N., Stamminger, T., and Orr, A. (2006). PML contributes to a cellular mechanism of repression of herpes simplex virus type 1 infection that is inactivated by ICP0. J. Virol. 80, 7995-8005.

Forde, J. E., and Dale, T. C. (2007). Glycogen synthase kinase 3: a key regulator of cellular fate. Cell. Mol. Life Sci. 64, 1930-1944.

Friborg, J., Kong, W., Hottiger, M. O., and Nabel, G. J. (1999). p53 inhibition by the LANA protein of KSHV protects against cell death. Nature 402, 889-894.

Fu, C., Ahmed, K., Ding, H., Ding, X., Lan, J., Yang, Z., Miao, Y., Zhu, Y., Shi, Y., Zhu, J., Huang, H., and Yao, X. (2005). Stabilization of PML nuclear localization by conjugation and oligomerization of SUMO-3. Oncogene 24, 5401-5413.

Fujimuro, M., and Hayward, S. D. (2003). The latency-associated nuclear antigen of Kaposi's sarcoma-associated herpesvirus manipulates the activity of glycogen synthase kinase-3 beta. J. Virol. 77, 8019-8030.

Fujimuro, M., Liu, J., Zhu, J., Yokosawa, H., and Hayward, S. D. (2005). Regulation of the interaction between glycogen synthase kinase 3 and the Kaposi's sarcoma-associated herpesvirus latency-associated nuclear antigen. J. Virol. 79, 10429-10441.

Ganem, D. (2010). KSHV and the pathogenesis of Kaposi sarcoma: listening to human biology and medicine. J. Clin. Invest. 120, 939-949.

Geiss-Friedlander, R., and Melchior, F. (2007). Concepts in sumoylation: a decade on. Nat. Rev. Mol. Cell Biol. 8, 947-956.

Girdwood, D., Bumpass, D., Vaughan, O. A., Thain, A., Anderson, L. A., Snowden, A. W., Garcia-Wilson, E., Perkins, N. D., and Hay, R. T. (2003). P300 transcriptional repression is mediated by SUMO modification. Mol. Cell 11, 1043-1054.

Goldberg, A. D., Banaszynski, L. A., Noh, K. M., Lewis, P. W., Elsaesser, S. J., Stadler, S., Dewell, S., Law, M., Guo, X., Li, X., Wen, D., Chapgier, A., DeKelver, R. C., Miller, J. C., Lee, Y. L., Boydston, E. A., Holmes, M. C., Gregory, P. D., Greally, J. M., Rafii, S., Yang, C., Scambler, P. J., Garrick, D., Gibbons, R. J., Higgs, D. R., Cristea, I. M., Urnov, F. D., Zheng, D., and
Allis, C. D. (2010). Distinct factors control histone variant H3.3 localization at specific genomic regions. Cell 140, 678-691.

Goodarzi, A. A., Kurka, T., and Jeggo, P. A. (2011). KAP-1 phosphorylation regulates $\mathrm{CHD} 3$ nucleosome remodeling during DNA doublestrand break response. Nat. Struct. Mol. Biol. 18, 831-839.

Gwack, Y., Nakamura, H., Lee, S. W., Souvis, J., Yustein, J. T., Gyri, S., Kung, H. J., and Jung, J. U. (2003). Poly(ADP-ribose) polymerase 1 and Ste20-like kinase hKFC act as transcriptional repressors for gamma2 herpesvirus lytic replication. Mol. Cell. Biol. 23, 8282-8294.

Hagen, T. (2009). Characterization of the interaction between latencyassociated nuclear antigen and glycogen synthase kinase 3 beta. $J$. Virol. 83, 6312-6317.

Heaphy, C. M., de Wilde, R. F., Jiao, Y., Klein, A. P., Edil, B. H., Shi, C., Bettegowda, C., Rodriguez, F. J., Eberhart, C. G., Hebbar, S., Offerhaus, G. J., McLendon, R., Rasheed, B. A., He, Y., Yan, H., Bigner, D. D., Oba-Shinjo, S. M., Marie, S. K., Riggins, G. J., Kinzler, K. W., Vogelstein, B., Hruban, R. H., Maitra, A., Papadopoulos, N., and Meeker, A. K. (2011). Altered telomeres in tumors with ATRX and DAXX mutations. Science 333, 425.

Hofmann, H., Floss, S., and Stamminger, T. (2000). Covalent modification of the transactivator protein IE2-p86 of human cytomegalovirus by conjugation to the ubiquitinhomologous proteins SUMO-1 and hSMT3b. J. Virol. 74, 2510-2524.

Hsu, C. H., Chang, M. D., Tai, K. Y., Yang, Y. T., Wang, P. S., Chen, C. J., Wang, Y. H., Lee, S. C., Wu, C. W., and Juan, L. J. (2004). HCMV IE2mediated inhibition of HAT activity downregulates p53 function. EMBO J. 23, 2269-2280.

Hu, J., Garber, A. C., and Renne, R. (2002). The latency-associated nuclear antigen of Kaposi's sarcomaassociated herpesvirus supports latent DNA replication in dividing cells. J. Virol. 76, 11677-11687.

Hwang, S., Gwack, Y., Byun, H., Lim, C., and Choe, J. (2001). The Kaposi's sarcoma-associated herpesvirus $\mathrm{K} 8$ protein interacts with CREB-binding protein (CBP) and represses CBP-mediated transcription. J. Virol. 75, 9509-9516.

Ishov, A. M., Sotnikov, A. G., Negorev, D., Vladimirova, O. V., Neff, N., Kamitani, T., Yeh, E. T., Strauss, J. F., 3rd, and Maul, G. G. (1999). PML is critical for ND10 formation and recruits the PML-interacting protein Daxx to this nuclear structure when modified by SUMO-1. J. Cell Biol. 147, 221-234.

Izumiya, Y., Ellison, T. J., Yeh, E. T., Jung, J. U., Luciw, P. A., and Kung, H. J. (2005). Kaposi's sarcoma-associated herpesvirus K-bZIP represses gene transcription via SUMO modification. J. Virol. 79, 9912-9925.

Izumiya, Y., Izumiya, C., Van Geelen, A., Wang, D. H., Lam, K. S., Luciw, P. A., and Kung, H. J. (2007). Kaposi's sarcoma-associated herpesvirus-encoded protein kinase and its interaction with K-bZIP. $J$. Virol. 81, 1072-1082.

Kedes, D. H., Lagunoff, M., Renne, R., and Ganem, D. (1997). Identification of the gene encoding the major latency-associated nuclear antigen of the Kaposi's sarcoma-associated herpesvirus. J. Clin. Invest. 100, 2606-2610.

Kelley-Clarke, B., De Leon-Vazquez, E., Slain, K., Barbera, A. J., and Kaye, K. M. (2009). Role of Kaposi's sarcoma-associated herpesvirus Cterminal LANA chromosome binding in episome persistence. J. Virol. 83, 4326-4337.

Kodadek, T., Sikder, D., and Nalley, K. (2006). Keeping transcriptional activators under control. Cell 127, 261-264.

Komatsu, T., Ballestas, M. E., Barbera, A. J., Kelley-Clarke, B., and Kaye, K. M. (2004). KSHV LANA1 binds DNA as an oligmer and residues $\mathrm{N}$-terminal to the oligomerization domain are essential for DNA binding, replication, and episome persistence. Virology 319, 225-336.

Krishnakumar, R., and Kraus, W. L. (2010). The PARP side of the nucleus: molecular actions, physiological outcomes, and clinical targets. Mol. Cell 39, 8-24.

Lan, K., Kuppers, D. A., and Robertson, E. S. (2005). Kaposi's sarcomaassociated herpesvirus reactivation is regulated by interaction of latency-associated nuclear antigen with recombination signal sequence-binding protein J-kappa, the major downstream effector of the Notch signaling pathway. $J$. Virol. 79, 3468-3478.

Ledl, A., Schmidt, D., and Muller S. (2005). Viral oncoproteins E1A and $\mathrm{E} 7$ and cellular $\mathrm{LxCxE}$ proteins repress SUMO modification of the retinoblastoma tumor suppressor. Oncogene 24, 3810-3818.

Lefort, S., Gravel, A., and Flamand, L. (2010). Repression of interferonalpha stimulated genes expression by Kaposi's sarcoma-assocaited herpesvirus K-bZIP protein. Virology 408, 14-30.

Lewis, P. W., Elsaesser, S. J., Noh, K. M., Stadler, S. C., and Allis, C. D. (2010). Daxx is an H3.3-specific histone chaperone and cooperates with ATRX in replication-independent chromatin assembly at telomeres. Proc. Natl. Acad. Sci. U.S.A. 107, 14075-14080.

Lilley, C. E., Schwartz, R. A., and Weitzman, M. D. (2007). Using or abusing: viruses and the cellular DNA damage response. Trends Microbiol. 15, 119-126.

Lim, C., Gwack, Y., Hwang, S., Kim, S., and Choe, J. (2001). The transcriptional activity of cAMP rsponse element-binding protein is modulated by the latency associated nuclear antigen of Kaposi's sarcomaassociated herpesvirus. J. Biol. Chem. 276, 31016-31022.

Lin, D. Y., Huang, Y. S., Jeng, J. C., Kuo, H. Y., Chang, C. C., Chao, T. T., Ho, C. C., Chen, Y. C., Lin, T. P., Fang, H. I., Hung, C. C., Suen, C. S., Hwang, M. J., Chang, K. S., Maul, G. G., and Shih, H. M. (2006). Role of SUMOinteracting motif in Daxx SUMO modification, subnuclear localization, and repression of sumoylated transcription factors. Mol. Cell 24, 341-354

Lin, S. F., Robinson, D. R., Miller, G., and Kung, H. J. (1999). Kaposi's sarcoma-associated herpesvirus encodes a bZIP protein with homology to BZLF1 of Epstein-Barr virus. J. Virol. 73, 1909-1917.

Liu, J., Martin, H., Shamay, M., Woodard, C., Tang, Q.-Q., and Hayward, S. D. (2007). Kaposi's sarcoma-associated herpesvirus LANA protein downregulates nuclear glycogen synthase kinase 3 activity and consequently blocks differentiation. J. Virol. 81, 4722-4731.

Lu, F., Day, L., Gao, S. J., and Lieberman, P. M. (2006). Acetylation of the latency-associated nuclear antigen regulates repression of Kaposi's sarcoma-associated herpesvirus lytic transcription. J. Virol. 80, 5273-5282.

Lukac, D. M., Kirshner, J. R., and Ganem, D. (1999). Transcriptional activation by the product of open reading frame 50 of Kaposi's sarcoma-associated herpesvirus is required for lytic viral reactivation in B cells. J. Virol. 73, 9348-9361.

Lukac, D. M., Renne, R., Kirshner, J. R., and Ganem, D. (1998). Reactivation of Kaposi's sarcoma-associated herpesvirus infection from latency 
by expression of the ORF 50 transactivator, a homolog of the EBV R protein. Virology 252, 304-312.

Lukashchuk, V., McFarlane, S., Everett, R. D., and Preston, C. M. (2006). Human cytomegalovirus protein pp71 displaces the chromatinassociated factor ATRX from nuclear domain 10 at early stages of infection. J. Virol. 82, 12543-12554.

Marcos-Villar, L., Lopitz-Otsoa, F., Gallego, P., Munoz-Fontela, C., Gonzalez-Santamaria, J., Campagna, M., Shou-Jiang, G., Rodriguez, M. S., and Rivas, C. (2009). Kaposi's sarcoma-associated herpesvirus protein LANA2 disrupts PML oncogenic domains and inhibits PML-mediated transcriptional repression of the survivin gene. $J$. Virol. 83, 8849-8858.

Mesri, E. A., Cesarman, E., and Boshoff, C. (2010). Kaposi's sarcoma and its associated herpesvirus. Nat. Rev. Cancer 10, 707-719.

Murakami, Y., Yamagoe, S., Noguchi, K., Takebe, Y., Takahashi, N., Uehara, Y., and Fukazawa, H. (2006). Ets-1-dependent expression of vascular endothelial growth factor receptors is activated by latency-associated nuclear antigen of Kaposi's sarcoma-associated herpesvirus through interaction with Daxx. J. Biol. Chem. 281, 28113-28121.

Muratani, M., and Tansey, W. P. (2003). How the ubiquitin-proteasome system controls transcription. Nat. Rev. Mol. Cell Biol. 4, 192-201.

Nagai, S., Davoodi, N., and Gasser, S. M. (2011). Nuclear organization in genome stability: SUMO connections. Cell Res. 21, 474-485.

Nakamura, H., Lu, M., Gwack, Y., Souvlis, J., Zeichner, S. L., and Jung, J. U. (2003). Global changes in Kaposi's sarcoma-associated virus gene expression patterns following expression of a tetracyclineinducible Rta transactivator. J. Virol. 77, 4205-4220.

Nathan, D., Ingvarsdottir, K., Sterner, D. E., Bylebyl, G. R., Dokmanovic, M., Dorsey, J. A., Whelan, K. A., Krsmanovic, M., Lane, W. S., Meluh, P. B., Johnson, E. S., and Berger, S. L. (2006). Histone sumoylation is a negative regulator in $\mathrm{Sac}$ charomyces cerevisiae and shows dynamic interplay with positiveacting histone modifications. Genes Dev. 20, 966-976.

Ohsaki, E., Ueda, K., Sakakibara, S., Do, E., Yada, K., and Yamanishi, K. (2004). Poly(ADP-ribose) polymerase 1 binds to Kaposi's sarcoma-associated herpesvirus
(KSHV) terminal repeat sequence and modulates KSHV replication in latency. J. Virol. 78, 9936-9946.

Platt, G. M., Simpson, G. R., Mittnacht, S., and Schulz, T. F. (1999). Latent nuclear antigen of Kaposi's sarcomaassociated herpesvirus interacts with RING3, a homolog of the Drosophila female sterile homeotic (fsh) gene. J. Virol. 73, 9789-9795.

Polson, A. G., Huang, L., Lukac, D. M., Blethrow, J. D., Morgan, D. O., Burlingame, A. L., and Ganem, D. (2001). Kaposi's sarcoma-associated herpesvirus $\mathrm{K}$ bZIP protein is phosphorylated by cyclin-dependent kinases. J. Virol. 75, 3175-3184.

Prudden, J., Pebernard, S., Raffa, G., Slavin, D. A., Perry, J. J., Tainer, J. A., McGowan, C. H., and Boddy, M. N. (2007). SUMO-targeted ubiquitin ligases in genome stability. $E M B O \mathrm{~J}$. 26, 4089-4101.

Radkov, S. A., Kellam, P., and Boshoff, C. (2000). The latent nuclear antigen of Kaposi sarcoma-associated herpesvirus targets the retinoblastomaE2F pathway and with the oncogene Hras transforms primary rat cells. Nat. Med. 6, 1121-1127.

Rosas-Acosta, G., Langereis, M. A., Deyrieux, A., and Wilson, V. G. (2005). Proteins of the PIAS family enhance the sumoylation of the papillomavirus E1 protein. Virology 331, 190-203.

Rouleau, M., Patel, A., Hendzel, M. J., Kaufmann, S. H., and Poirier, G. G. (2010). PARP inhibition: PARP1 and beyond. Nat. Rev. Cancer 10, 293-301.

Schulz, T. F. (2001). KSHV/HHV8assoicated lymphoproliferations in the AIDS setting. Eur. J. Cancer 37, 1217-1226.

Seeler, J. S., Marchio, A., Losson, R., Desterro, J. M., Hay, R. T., Chambon, P., and Dejean, A. (2001). Common properties of nuclear body protein SP100 and TIFlalpha chromatin factor: role of SUMO modification. Mol. Cell. Biol. 21, 3314-3324.

Song, M. J., Li, X., Brown, H. J., and Sun, R. (2002). Characterization of interactions between RTA and the promoter of polyadenylated nuclear RNA in Kaposi's sarcoma-associated herpesvirus/human herpesvirus 8. J. Virol. 76, 5000-5013.

Spengler, M. L., Kurapatwinski, K., Black, A. R., and Azizkhan-Clifford, J. (2002). SUMO-1 modification of human cytomegalovirus IE1/IE72. J. Virol. 76, 2990-2996.

Stielow, B., Sapetschnig, A., Wink, C., Kruger, I., and Suske, G. (2008).
SUMO-modified Sp3 represses transcription by provoking local heterochromatic gene silencing. $E M B O$ Rep. 9, 899-906.

Sun, H., Leverson, J. D., and Hunter, T. (2007). Conserved function of RNF4 family proteins in eukaryotes: targeting a ubiquitin ligase to SUMOylated proteins. EMBO J. 26 , 4102-4112.

Sun, R., Lin, S. F., Gradoville, L., Yuan, Y., Zhu, F., and Miller, G. (1998). A vira gene that activates lytic cycle expression of Kaposi's sarcoma-associated herpesvirus. Proc. Natl. Acad. Sci. U.S.A. 95, 10866-10871.

Tassi, E., Biesova, Z., Di Fiore, P. P., Gutkind, J. S., and Wong, W. T. (1999). Human JIK, a novel member of the STE20 kinas family that inhibits JNK and is negatively regulated by epidermal growth factor. $J$. Biol. Chem. 274, 33287-33295.

Tatham, M. H., Geoffroy, M. C., Shen, L., Plechanovova, A., Hattersley, N. Jaffray, E. G., Palvimo, J. J., and Hay, R. T. (2008). RNF4 is a polySUMO-specific E3 ubiquitin ligase required for arsenic-induced PML degradation. Nat. Cell Biol. 10, 538-546.

Tavalai, N., Papior, P., Rechter, S., Leis, M., and Stamminger, T. (2006). Evidence for a role of the cellular ND10 protein PML in mediating intrinsic immunity against human cytomegalovirus infections. J. Virol. 80, 8006-8018.

Tempera, I., Deng, Z., Atanasiu, C. Chen, C.-J., D'Erme, M., and Lieberman, P. (2010). Regulation of Epstein-Barr virus OriP replication by poly(ADP-ribose) polymerase 1 . J. Virol. 84, 4988-4997.

Uchimura, Y., Ichimura, T., Uwada, J., Tachibana, T., Sugahara, S., Nakao, M., and Saitoh, H. (2006). Involvement of SUMO modification in MBD1- and MCAF1-mediated heterochromatin formation. J. Biol Chem. 281, 23180-23190.

Uzunova, K., Gottsche, K., Miteva, M., Weisshaar, S. R., Glanemann, C., Schnellhardt, M., Niessen, M. Scheel, H., Hofmann, K., Johnson, E. S., Praefcke, G. J., and Dohmen, R. J. (2007). Ubiquitin-dependent proteolytic control of SUMO conjugates. J. Biol. Chem. 282, 34167-34175.

Varjosalo, M., Bjorklund, M., Cheng, F., Synanen, H., Kivioja, T., Kilpinen, S., Sun, Z., Kallioniemi, O., Stunnenberg, H. G., He, W.-W., Ojala, P., and Taipale, J. (2008). Application of active and kinase-deficient kinome collection for identification of kinases regulating hedgehog signaling. Cell 133, 537-548.
Verma, S. C., Borah, S., and Robertson, E. (2004). Latency-associated nuclear antigen of Kaposi's sarcomaassociated herpesvirus upregulates transcription of human telomerase reverse transcriptase promoter through interaction with transcription factor Sp1. J. Virol. 78, 10348-10359.

Verma, S. C., Lan, K., and Robertson, E. (2007). Structure and function of latency-associated nuclear antigen. Curr. Top. Microbiol. Immunol. 312, 101-136.

Weitzman, M. D., Lilley, C. E., and Chaurushiya, M. S. (2010). Genomes in conflict: maintaining genome integrity during virus infection. Annu. Rev. Microbiol. 64, 61-81.

West, J. T., and Wood, C. (2003). The role of Kaposi's sarcomaassociated herpesvirus/human herpesvirus-8 regulator of transcription activation (RTA) in control of gene expression. Oncogene 22, 5150-5163.

Wu, H., Sun, L., Zhang, Y., Chen, Y., Shi, B., Li, R., Wang, Y., Liang, J., Fan, D. Wu, G., Wang, D., Li, S., and Shang, Y. (2006). Coordinated regulation of AIB1 transcriptional activity by sumoylation and phosphorylation. J. Biol. Chem. 281, 21848-21856.

Wu, Y. C., Deyrieux, A. F., and Wilson, V. G. (2007). Papillomaviruses and the host SUMOylation system. Biochem. Soc. Trans. 35, 1433-1435.

Xie, Y., Kerscher, O., Kroetz, M. B., McConchie, H. F., Sung, P., and Hochstrasser, M. (2007). The yeast Hex3.Slx8 heterodimer is a ubiquitin ligase stimulated by substrate sumoylation. J. Biol. Chem. 282, 34176-34184.

Xu, Y., AuCoin, D. P., Huete, A. R., Cei, S. A., Hanson, L. J., and Pari, G. S. (2005). A Kaposi's sarcomaassociated herpesvirus/human herpesvirus 8 ORF50 deletion mutant is defective for reactivation of latent virus and DNA replication. J. Virol. 79, 3479-3487.

Yang, Z., Yan, Z., and Wood, C. (2008). Kaposi's sarcoma-associated herpesvirus transactivator RTA promotes degradation of the repressors to regulate viral lytic replication. $J$. Virol. 82, 3590-3603.

Yu, Y., Wang, S. E., and Hayward, G. S. (2005). The KSHV immediateearly transcription factor RTA encodes ubiquitin E3 ligase activity that targets IRF7 for proteasomemediated degradation. Immunity 22 , 59-70.

Yustein, J. T., Li, D., Robinson, D., and Kung, H. J. (2000). KFC, a Ste20-like kinase with mitogenic potential and 
capability to activate the SAPK/JNK pathway. Oncogene 19, 710-718.

Yustein, J. T., Xia, L., Kahlenburg, J. M., Robinson, D., Templeton, D., and Kung, H. J. (2003). Comparative studies of a new subfamily of human Ste-20-like kinases: homodimerization, subcellular localization, and selective activation of MKK3 and p38. Oncogene 22, 6129-6141.

Zhong, S., Muller, S., Ronchetti, S., Freemont, P. S., Dejean, A., and Pandolfi, P. P. (2000). Role of SUMO-1-modified PML in nuclear body formation. Blood 95, 2748-2752.

Zimnik, S., Gaestel, M., and Niedenthal, R. (2009). Mutually exclusive STAT1 modifications identified by Ubc9/substrate dimerizationdependent SUMOylation. Nucleic Acids Res. 37, e30.

Conflict of Interest Statement: The authors declare that the research was conducted in the absence of any commercial or financial relationships that could be construed as a potential conflict of interest.

Received: 22 November 2011; paper pending published: 12 December 2011; accepted: 18 January 2012; published online: 14 February 2012.

Citation: Campbell $M$ and Izumiya $Y$ (2012) Post-translational modifications of Kaposi's sarcoma-associated herpesvirus regulatory proteins - SUMO and KSHV. Front. Microbio. 3:31. doi: 10.3389/fmicb.2012.00031

This article was submitted to Frontiers in Virology, a specialty of Frontiers in Microbiology.

Copyright (C) 2012 Campbell and Izumiya. This is an open-access article distributed under the terms of the Creative Commons Attribution Non Commercial License, which permits noncommercial use, distribution, and reproduction in other forums, provided the original authors and source are credited. 